Assiut University web-site: $\underline{w w w . a u n} . e d u . e g$

\title{
CONSEQUENCES OF HEPATORENAL TOXICITY INDUCED BY GENTAMICIN IN CHICKENS
}

\author{
NAGAH ARAFAT ${ }^{1}$; REHAM EL-SHAFEI ${ }^{2}$; WALAA AWADIN ${ }^{3}$; VERGINIA FARAG ${ }^{4}$ and \\ RASHA SALEH ${ }^{5}$ \\ ${ }^{1}$ Department of Poultry Diseases, Faculty of Veterinary Medicine, Mansoura University, Egypt \\ ${ }^{2}$ Department of Pharmacology, Faculty of Veterinary Medicine, Mansoura University, Egypt \\ ${ }^{3}$ Department of Pathology, Faculty of Veterinary Medicine, Mansoura University, Egypt \\ ${ }^{4}$ Department of Clinical Pathology Faculty of Veterinary Medicine, Mansoura University, Egypt \\ ${ }^{5}$ Department of Physiology, Faculty of Veterinary Medicine, Mansoura University, Egypt
}

Received: 28 June 2017; $\quad$ Accepted: 30 July 2017

\begin{abstract}
Gentamicin, one of aminoglycosides, was greatly used in treatment of different bacterial infections in animal. This study investigated the hepato-nephrotoxicity induced by single intramuscular (IM) injection gentamicin in chickens. A total number of 90, one day-old, Balady chicks were randomly divided into 3 groups each with 30 birds. Group (1) served as a control; Group (2) was given $25 \mathrm{mg}$ gentamicin $/ \mathrm{kg}$ body weight (BW), Group (3) was given $50 \mathrm{mg}$ gentamicin $/ \mathrm{kg} \mathrm{BW}$. Hepato-nephrotoxicity was evaluated by measuring growth performances, mortality rats, hematological parameters, biochemical markers; reduced glutathione (GSH), nitric oxide (NO), liver function tests, serum urea and creatinine levels, as well as, by evaluation of histopathological pictures of liver and kidney. $50 \mathrm{mg}$ gentamicin $/ \mathrm{kg}$ BW reduced growth performance and decreased red blood cell (RBC) count, hemoglobin $(\mathrm{Hb})$ concentration, mean corpuscular hemoglobin concentration (MCHC), total protein, albumin, globulin levels and albumin/globulin (A/G) ratio. There were significant heterophilic leukocytosis and eosinophilia. Levels of alkaline phosphatase (ALP), aspartate aminotransferase (AST) and alanine aminotransferase (ALT), urea, creatinine, liver and kidney NO were increased, meanwhile levels of GSH were decreased. Severe congestion, hemorrhage and multifocal coagulative necrotic areas were microscopically detected in liver and kidneys. Gentamicin injection altered hematological, biochemical and serum antioxidants pictures and induced hepatic and renal lesions in a dose dependent manner. The chickens administered $50 \mathrm{mg} / \mathrm{kg}$ BW gentamicin were more severely affected than $25 \mathrm{mg} / \mathrm{kg} \mathrm{BW}$ gentamicin.
\end{abstract}

Key words: Antioxidant, gentamicin, growth performance, hematology, histopathology.

\section{INTRODUCTION}

Aminoglycosides are commonly used to treat bacterial infections because of their low cost and limited antibiotic resistance. Gentamicin, one of aminoglycosides, is greatly used in treatment of different bacterial infections in both animal and human (Kang et al., 2013).

It is used on a large scale in poultry farm, which is active against different Gram negative and some gram positive bacteria. Gentamicin was given to poultry by intramuscular (IM) injection due to its poor absorption from digestive tract (Haritova et al., 2004).

Corresponding author: Dr. REHAM EL-SHAFEI

E-mail address:Dr_reham16@yahoo.com

Present address: Department of Pharmacology, Faculty of Veterinary Medicine, Mansoura University, Egypt.
In field cases of gentamicin toxicity of White Leghorn layer flocks, dehydration, emaciation, watery diarrhea, increased water intake and high mortality were clinically observed (Islam et al., 2011).

Experimentally, pathological effects of gentamicin have been reported in growing cockerels (Khan et al., 2008) and growing broilers (Javed et al., 2008). Those studies reported the safe dose of gentamicin as $10 \mathrm{mg} / \mathrm{kg}$ body weight (BW) in day old broilers (Saleemi et al., 2009) and in growing White Leghorn cockerels (Khan et al., 2008). Generally, gentamicin as all aminoglycosides could induce tubular nephrotoxicity (Ravi et al., 2015).

Earlier studies found that gentamicin could release iron from renal mitochondria and form an iron gentamicin complex that promotes the production of free radicals and reactive oxygen species (ROS) (Kovacset, 2012). ROS and increased oxidative stress appear to be implicated in the pathophysiology of 
gentamicin induced nephrotoxicity (Morales et al., 2010). Furthermore, reduced glutathione content is reduced in renal cortex (Sener et al., 2002).

Several studies showed that ROS involved in gentamicin -induced nephrotoxicity, as well as, it has been shown to enhance the generation of superoxide anion $(\mathrm{O} 2)$, peroxynitriteanion (ONOO), hydrogen peroxide $(\mathrm{H} 2 \mathrm{O} 2)$, and hydroxyl radical $(\mathrm{OH})$ from renal cortical mitochondria, which is responsible for increase lipid peroxidation and decrease antioxidant enzymes (Tavafi and Ahmad, 2011).

Therefore, the aim of this study was to compare the effect of two different doses of gentamicin (25\&50 $\mathrm{mg} / \mathrm{kg} \mathrm{BW}$ as a single IM injection) on growth performance and pathophysiological picture in chickens.

\section{MATERIALS AND METHODS}

\section{Experimental Chickens and management}

A total number of 90 , one day-old, Balady chicks were maintained in cages, kept in well ventilated disinfected room under 24 hours lighting and were provided with a commercial starter, balanced ration free from any medication. The feed and water were offered ad-libitum to all birds throughout the experimental period. Animal procedures were performed in accordance with the recommendations of the 'Guide for the Care and Use of Laboratory Animals' approved by the Ethics Committee of the Faculty of Veterinary Medicine, Mansoura University.

\section{Drug (Gentamicin)}

Ato-Gent $10 \%{ }^{\circledR}$ (Injectable solution: each $1 \mathrm{ml}$ contains gentamicin sulfate $156.25 \mathrm{mg}$ equivalent gentamicin base $100 \mathrm{mg}$, Atcopharma, Egypt. Registration No 2265/2004, Batch No 419202. It was administrated on $21^{\text {st }}$ day of age at the rate of 25 and $50 \mathrm{mg} / \mathrm{kg} \mathrm{BW}$ as a single IM injection in pectoral muscles.

\section{Experimental design}

The 90 Balady chicks were randomly divided into 3 groups, each with 30 birds of two replicate. The treatment regimen was: Group (1) was served as control negative (injected with normal saline in pectoral muscles). Group (2) was given gentamicin $25 \mathrm{mg} / \mathrm{kg} \mathrm{BW}$ as a single IM injection in pectoral muscles. Group (3) was given a single IM injection of gentamicin $50 \mathrm{mg} / \mathrm{kg} \mathrm{BW}$ in pectoral muscles (Javed et al., 2013).

\section{Sampling:}

Blood samples were randomly gathered from the wing veins of five birds at the end of $1^{\text {st }}$ day, $3^{\text {rd }}$ day, $1^{\text {st }}$ week and $3^{\text {rd }}$ weeks post gentamicin injection, and were immediately divided into two separate tubes.
One of them was left in a plain test tube at room temperature for 1 hour and then centrifuged for 10 minutes at $3000 \mathrm{~g}$ to obtain the serum. The clear serum was stored in epindorf tubes at $-20^{\circ} \mathrm{C}$ for subsequent biochemical analysis. The other blood sample was collected with ethylene diamine tetra acetic acid (EDTA) (0.5 $\mathrm{mg} / \mathrm{ml}$ blood) for hematological examination.

Random 3 birds from each group were sacrificed at $1^{\text {st }}$ and $3^{\text {rd }}$ weeks post gentamicin injection. Each bird was weighed prior to killing and tissue specimens from liver and kidney were collected then washed with ice-cold normal saline. Slices from liver and kidney were used to prepare tissue homogenates (10\% weight/volume) in phosphate buffered saline ( $\mathrm{pH}$ 7.4) using ultrasonic homogenizer (Cole-Parmer Instrument Company, USA). Then, the homogenates were centrifuged (4000 round per minute for 15 minutes at $4^{\circ} \mathrm{C}$ ) and the supernatant was preserved at $-80^{\circ} \mathrm{C}$ until used for antioxidant analysis. The remaining specimens were fixed in $10 \%$ buffered formaldehyde for histopathological examination.

\section{Assessment of growth performance}

Birds were weighed and feed intake per each group was measured weekly. Feed intake was determined for each group as the distinction between food supplied and the remaining food toward the end of every week. BW gain was figured as the difference between the final and the initial weight of the bird. FCR was calculated by dividing the amount of consumed feed (g) during the week by the gain in BW (g) during the same week (Smith, 1999).

\section{Relative organ weights}

After killing, visceral organs (Liver, kidney, heart and spleen) were weighed and their relative weights were calculated as organ weight/ $\mathrm{BW} \times 100$.

\section{Clinical signs, mortalities and gross lesions}

The birds were watched twice daily for three weeks post gentamicin injection until the end of the study for recording clinical signs and mortality. Gross lesions on different visceral organs were recorded for killed and dead birds.

\section{Hematological analysis:}

Red blood cells (RBC) and total leukocytic count (TLC) were manually performed according to Feldman et al. (2002) using improved neubauer haemocytometer Natt and Herrick solution according to Natt and Herrick (1952). Hemoglobin (Hb) concentration estimated spectrophotometrically next centrifugation using the cyanomethaemoglobin method, according to Dein (1984). Packed cell volume (PCV), mean corpuscular volume (MCV) (fL), mean corpuscular hemoglobin $(\mathrm{MCH})(\mathrm{pg})$ and mean corpuscular hemoglobin concentration (MCHC) (\%) were calculated by standard formula according to 
Feldman et al. (2002). The differential leukocytic count was made immediately by manual method (Andreasen and Latimer, 1990).

\section{Serum biochemical analysis:}

Frozen serum samples were subjected for estimation of alkaline phosphatase (ALP) (El tech, France), aspartate aminotransferase (AST) and alanine aminotransferase (ALT) (Randox Co., UK), creatinine (Human, Germany), urea (Diamond, Egypt), total protein and albumin (Stanbio, USA). Globulin and albumin/globulin (A/G) ratio were calculated according (Kaneko et al., 1997).

\section{Antioxidant analysis}

Nitric oxide (NO) and reduced glutathione (GSH) were determined by enzymatic colorimetric method in liver and kidney extract using readymade kits provided by Bio-diagnostic, Egypt. The NO was estimated (Ignarro et al., 1987) based on the presence of nitrite in acid medium, the formed product was coupled with $\mathrm{N}$-(1-naphthyl) ethylenediamine. GSH estimation was done according to the reduction of 5 , 5 dithiobis (2-nitrobenzoic acid) (DTNB) with the glutathione producing a yellow product (Beutler et al., 1963).

\section{Histopathology:}

Liver and kidneys were collected from each bird during postmortem examination, fixed in $10 \%$ neutral buffered formalin and processed for histopathological examination by paraffin embedding method. Five $\mu \mathrm{m}$ thickness sections were cut and stained with hematoxylin and eosin according to Bancroft and Gamble, (2007).

\section{Statistical analysis:}

The data were statistically analyzed using SAS 9.2 for windows (SAS, 2004). The tests were used are oneway analysis of variance (ANOVA) and Duncan's multiple-range tests. They were done for all hematological, biochemical and antioxidant parameters. The data were expressed as mean \pm SD $(\mathrm{P}$ $\leq 0.05)$.

\section{Results}

\section{Growth performance}

The results showed that, growth performance parameters represented by mean body weight gain, average feed intake and FCR were significantly decreased in birds of both gentamicin treated Groups (2\&3) when compared with control Group (1). However, the deleterious effect of $25 \mathrm{mg} / \mathrm{kg} \mathrm{BW}$ gentamicin in Group (2) was less than $50 \mathrm{mg} / \mathrm{kg} \mathrm{BW}$ gentamicin in Group (3) (Table 1).

\section{Relative organs weight}

There were significant increase in relative weights of liver, kidneys, heart and spleen in both gentamicin treated Groups (2\&3) when compared with the control
Group (1) at $1^{\text {st }}$ and $3^{\text {rd }}$ weeks post gentamicin injection.

\section{Mortality rate, clinical signs and gross lesions}

No clinical signs were observed in birds of Group (1) along the experimental period. Meanwhile, birds in Group (2) were slightly depressed. Birds in Group (3) showed sever clinical signs including depression, watery diarrhea, increased water intake and emaciation as shown in (Table 3). No mortalities were recorded in birds of Groups (1\&2). No postmortem changes were found in Group (1) after $1^{\text {st }}$ and $3^{\text {rd }}$ weeks post gentamicin injection. Livers from dead and sacrificed birds after $1^{\text {st }}$ week post gentamicin injection showed varying degrees of congestion, friableness and swelling in Groups (2\&3) with ascending changes. At the $3^{\text {rd }}$ week post gentamicin injection, the liver changes were more pronounced in Group (3) than in Group (2). Severe congestion and swelling in kidneys with distension of ureters with urates were shown only in Group (3). Meanwhile, slightly congested kidneys were seen in birds of Group (2).

\section{Hematological results}

At $1^{\text {st }}$ and $3^{\text {rd }}$ weeks post gentamicin injection, RBC, $\mathrm{Hb}$ and $\mathrm{MCHC}$ of Groups (2\&3) were significantly decreased. Meanwhile, the MCV of Groups (2\&3) was significantly increased when compared with Group (1) (Table 4).

Regarding to the leukogram, there was a significant heterophilic leukocytosis in Groups $(2 \& 3)$ at $1^{\text {st }}$ and $3^{\text {rd }}$ weeks post gentamicin injection. However, lymphopenia was recorded in Group (3) at $3^{\text {rd }}$ week. Eosinophilia was significantly existed in the Group (3) only at $1^{\text {st }}$ week post gentamicin injection (Table 5).

\section{Biochemical results}

Significant increases of AST, ALP and creatinine levels and decreases of total protein in Group (3) were prominent at all periods of the experiment and in Group (2) at $3^{\text {rd }}$ week comparing with Group (1) as shown in (Table 6). In Groups (2\&3), significant decrease of globulin (at $1^{\text {st }}$ and $3^{\text {rd }}$ day) and $\mathrm{A} / \mathrm{G}$ ratio (at $1^{\text {st }}$ and $3^{\text {rd }}$ weeks post gentamicin injection) was observed comparing with Group (1). The urea level significantly increased in Groups (2\&3) along the experimental period. The creatinine level significantly increased Group (3) along the experimental period and in Group (2) at $3^{\text {rd }}$ day and $1^{\text {st }}$ and $2^{\text {nd }}$ week when compared with those of Group (1).

\section{Antioxidant results}

Significant increases of liver and kidney NO levels at $1^{\text {st }}$ and $3^{\text {rd }}$ weeks post gentamicin injection in Groups (2\&3) when compared with Group (1) (Table 7). Liver GSH level significantly decreased in Groups 
(2\&3) at $1^{\text {st }}$ and $3^{\text {rd }}$ weeks post gentamicin injection. Meanwhile, kidney GSH significantly decreased at $3^{\text {rd }}$ week only when compared with the Group (1).

\section{Histopathological examination:}

Liver showed normal shape and sizes of hepatocytes in Group (1) at two sacrifices. Liver from Group (2) at $1^{\text {st }}$ and $3^{\text {rd }}$ weeks post gentamicin injection showed vacuolar to hydropic degeneration, mild to severe congestion, perivascular edema and dilation of bile duct with hyperplasia of biliary epithelium. Few leukocytic cells infiltration was observed perivascular around hepatic blood vessels (Fig. 1). Liver from Group (3) at $1^{\text {st }}$ week post gentamicin injection showed similar lesions to those observed in Group (2). At $3^{\text {rd }}$ week post gentamicin injection, from Group (3) showed hemorrhage, perivascular fibrosis, new bile ductules formation and multifocal areas of coagulative necrosis in hepatic parenchyma (Fig. 2). Lymphocytic follicular aggregation was seen in the examined liver from all groups. However, the frequency and size of the follicles increased in liver from treated Groups $2 \& 3$ and in correspondence to duration of exposure (Fig. 3).

Kidneys showed normal tubules and glomeruli in Group (1) in two sacrifices. At $1^{\text {st }}$ week post gentamicin injection, kidneys from Groups (2\&3) showed hydropic degeneration with detachment of renal epithelium from basement membrane, shrunken glomeruli and interstitial lymphocytic cells infiltration (Fig. 4). Similar lesions were observed in kidneys from Groups $(2 \& 3)$ at $3^{\text {rd }}$ week post gentamicin injection with increased severity and distribution of lesions. Focal coagulative necrosis, congestion, perivascular edema, hemorrhage and degenerated glomeruli were additionally demonstrated in Group (3) at $3^{\text {rd }}$ week post gentamicin injection (Fig. 5).

Table 1: Mean weight gain, average feed intake and Average feed conversation rate post gentamicin injection.

\begin{tabular}{|c|c|c|c|c|}
\hline \multirow{2}{*}{ Weeks post } & \multirow{2}{*}{$\begin{array}{l}\text { Parameter } \\
\text { gentamicin } \\
\text { Injection }\end{array}$} & \multicolumn{3}{|c|}{ Groups } \\
\hline & & Group 1 & Group 2 & Group 3 \\
\hline \multirow{2}{*}{$\begin{array}{l}\text { Mean weight } \\
\text { gain }(\mathrm{g})\end{array}$} & 1 & $93.5 \pm 1.4^{\mathrm{a}}$ & $81.5 \pm 0.29^{b}$ & $65.5 \pm 0.69^{c}$ \\
\hline & 3 & $148.8 \pm 2.6^{\mathrm{a}}$ & $111.3 \pm 1.9^{b}$ & $85.7 \pm 1.1^{\mathrm{c}}$ \\
\hline \multirow{2}{*}{$\begin{array}{l}\text { Average feed } \\
\text { intake }(g)\end{array}$} & 1 & $388.9 \pm 24.6^{a}$ & $350.4 \pm 25.3^{b}$ & $301.6 \pm 21.1^{\mathrm{c}}$ \\
\hline & 3 & $492.8 \pm 38.6^{\mathrm{a}}$ & $444.1 \pm 35.3^{b}$ & $381.3 \pm 33.8^{c}$ \\
\hline \multirow{2}{*}{$\begin{array}{l}\text { Average feed } \\
\text { conversation rate (FCR) }\end{array}$} & 1 & $4.16 \pm 0.32^{\mathrm{c}}$ & $4.3 \pm 0.59^{\mathrm{b}}$ & $4.6 \pm 0.38^{\mathrm{a}}$ \\
\hline & 3 & $3.31 \pm 0.44^{\mathrm{c}}$ & $3.99 \pm 0.55^{\mathrm{b}}$ & $4.45 \pm 0.50^{\mathrm{a}}$ \\
\hline
\end{tabular}

Different letters within the same row were significantly difference at $\mathrm{P} \leq 0.05$.

Table 2: Relative organs weights post gentamicin injection.

\begin{tabular}{|c|c|c|c|c|}
\hline \multirow[t]{2}{*}{ Organ } & \multirow{2}{*}{$\begin{array}{l}\text { Weeks post } \\
\text { gentamicin } \\
\text { Injection }\end{array}$} & \multicolumn{3}{|c|}{ Groups } \\
\hline & & Group 1 & Group 2 & Group 3 \\
\hline \multirow[t]{2}{*}{ Liver } & 1 & $2.72 \pm 0.14^{\mathrm{c}}$ & $3.4 \pm 0.26^{\mathrm{b}}$ & $4.22 \pm 0.31^{\mathrm{a}}$ \\
\hline & 3 & $2.9 \pm 0.16^{\mathrm{c}}$ & $4.92 \pm 0.31^{\mathrm{b}}$ & $6.94 \pm 0.41^{\mathrm{a}}$ \\
\hline \multirow[t]{2}{*}{ Kidney } & 1 & $0.62 \pm 0.01^{\mathrm{d}}$ & $0.91 \pm 0.06^{\mathrm{b}}$ & $1.31 \pm 0.1^{\mathrm{a}}$ \\
\hline & 3 & $0.59 \pm 0.02^{\mathrm{d}}$ & $0.93 \pm 0.11^{\mathrm{b}}$ & $1.6 \pm 0.13^{\mathrm{a}}$ \\
\hline \multirow[t]{2}{*}{ Heart } & 1 & $0.32 \pm 0.03^{\mathrm{d}}$ & $0.55 \pm 0.03^{\mathrm{b}}$ & $0.69 \pm 0.01^{\mathrm{a}}$ \\
\hline & 3 & $0.31 \pm 0.02^{\mathrm{c}}$ & $0.51 \pm 0.04^{\mathrm{b}}$ & $0.82 \pm 0.02^{\mathrm{a}}$ \\
\hline \multirow[t]{2}{*}{ Spleen } & 1 & $0.08 \pm 0.01^{\mathrm{b}}$ & $0.095 \pm 0.01^{b}$ & $0.13 \pm 0.01^{\mathrm{a}}$ \\
\hline & 3 & $0.082 \pm 0.01^{\mathrm{a}}$ & $0.097 \pm 0.03^{b}$ & $0.14 \pm 0.05^{\mathrm{b}}$ \\
\hline
\end{tabular}

Different letters within the same row were significantly difference at $(\mathrm{P} \leq 0.05)$. 
Table 3: Mortality rate and clinical signs of different groups post Gentamicin injection.

\begin{tabular}{|c|c|c|c|c|}
\hline \multirow[t]{3}{*}{ Parameter } & \multirow{3}{*}{$\begin{array}{l}\text { Weeks post } \\
\text { Gentamicin } \\
\text { injection }\end{array}$} & \multicolumn{3}{|c|}{ Groups } \\
\hline & & 1 & 2 & 3 \\
\hline & & Control & $\begin{array}{c}\text { 25mg/kg } \\
\text { Gentamicin }\end{array}$ & $\begin{array}{c}\text { 50mg/kg } \\
\text { Gentamicin }\end{array}$ \\
\hline \multirow{2}{*}{$\begin{array}{l}\text { Numbers of } \\
\text { mortalities }\end{array}$} & 1 & 0 & 0 & 5 \\
\hline & 3 & 0 & 0 & 0 \\
\hline Number of dead/total numbers $(\%)$ & $1 \& 3$ & $\begin{array}{c}0 / 30 \\
(0.0)^{\mathrm{b}}\end{array}$ & $\begin{array}{l}0 / 30 \\
(0.0)^{b}\end{array}$ & $\begin{array}{c}5 / 30 \\
(16.6)^{\mathrm{a}}\end{array}$ \\
\hline \multirow[t]{2}{*}{ Numbers of birds with clinical signs* } & 1 & 0 & 2 & 10 \\
\hline & 3 & 0 & 0 & 0 \\
\hline $\begin{array}{l}\text { Number of diseased /total numbers } \\
(\%)\end{array}$ & $1 \& 3$ & $\begin{array}{l}0 / 30 \\
(0.0)^{b}\end{array}$ & $\begin{array}{l}2 / 30 \\
(6.6)^{b}\end{array}$ & $\begin{array}{c}10 / 30 \\
(33.3)^{\mathrm{a}}\end{array}$ \\
\hline
\end{tabular}

* Clinical signs are represented by depression, watery diarrhea, increased water intake, and emaciation.

Different letters within the same row were significantly difference at $(\mathrm{P} \leq 0.05)$.

Table 4: Erythrogram of different groups post gentamicin injection.

\begin{tabular}{|c|c|c|c|c|c|c|}
\hline Groups & $\begin{array}{c}\text { RBC } \\
\left(10^{6} / \mu 1\right)\end{array}$ & $\begin{array}{c}\mathrm{Hb} \\
(\mathrm{g} / \mathrm{dl})\end{array}$ & $\begin{array}{r}\mathrm{PCV} \\
(\%) \\
\end{array}$ & $\begin{array}{c}\text { MCV } \\
(\mathrm{fl})\end{array}$ & $\begin{array}{c}\mathrm{MCH} \\
(\mathrm{pg})\end{array}$ & $\begin{array}{c}\mathrm{MCHC} \\
(\%)\end{array}$ \\
\hline \multicolumn{7}{|c|}{ Week 1} \\
\hline Group 1 & $3.24 \pm 0.39^{\mathrm{a}}$ & $7.97 \pm 0.85^{\mathrm{a}}$ & $38.91 \pm 0.79^{a}$ & $121.31 \pm 16.88^{b}$ & $24.63 \pm 1.62^{\mathrm{a}}$ & $20.48 \pm 2.18^{\mathrm{a}}$ \\
\hline Group 2 & $2.28 \pm 0.38^{b}$ & $5.23 \pm 0.96^{b}$ & $33.76 \pm 1.27^{\mathrm{a}}$ & $150.55 \pm 24.75^{\mathrm{ab}}$ & $22.99 \pm 2.91^{\mathrm{a}}$ & $15.54 \pm 3.23^{b}$ \\
\hline Group 3 & $2.15 \pm 0.43^{b}$ & $5.56 \pm 0.95^{b}$ & $36.40 \pm 6.81^{\mathrm{a}}$ & $169.73 \pm 7.39^{\mathrm{a}}$ & $26.39 \pm 1.0^{\mathrm{a}}$ & $15.56 \pm 0.41^{b}$ \\
\hline \multicolumn{7}{|c|}{ Week 3} \\
\hline Group 1 & $3.30 \pm 0.36^{\mathrm{a}}$ & $7.49 \pm 0.54^{\mathrm{a}}$ & $39.45 \pm 2.53^{\mathrm{a}}$ & $120.04 \pm 7.43^{b}$ & $22.90 \pm 2.99^{\mathrm{a}}$ & $19.07 \pm 2.14^{\mathrm{a}}$ \\
\hline Group 2 & $2.28 \pm 0.37^{\mathrm{b}}$ & $5.62 \pm 0.92^{b}$ & $39.57 \pm 2.23^{\mathrm{a}}$ & $175.80 \pm 22.02^{\mathrm{a}}$ & $25.13 \pm 5.89^{\mathrm{a}}$ & $14.18 \pm 1.82^{b}$ \\
\hline Group 3 & $2.41 \pm 0.08^{b}$ & $5.25 \pm 0.50^{b}$ & $40.74 \pm 1.42^{\mathrm{a}}$ & $169.03 \pm 10.56^{\mathrm{a}}$ & $21.80 \pm 2.76^{\mathrm{a}}$ & $12.88 \pm 1.16^{b}$ \\
\hline
\end{tabular}

Means with different superscript letters $(a, b)$ in the same vertical column are significantly different at $p \leq 0.05$.

Table 5: Leukogram of different groups post gentamicin injection.

\begin{tabular}{|c|c|c|c|c|c|c|}
\hline Groups & $\begin{array}{c}\text { TLC } \\
10^{3} / \mu 1\end{array}$ & $\begin{array}{c}\text { Lymphocytes } \\
10^{3} / \mu 1\end{array}$ & $\begin{array}{c}\text { Heterophils } \\
10^{3} / \mu \mathrm{l}\end{array}$ & $\begin{array}{c}\text { Monocytes } \\
10^{3} / \mu 1\end{array}$ & $\begin{array}{c}\text { Eosinophils } \\
10^{3} / \mu 1\end{array}$ & $\begin{array}{c}\text { Basophils } \\
10^{3} / \mu 1\end{array}$ \\
\hline \multicolumn{7}{|c|}{ Week 1} \\
\hline Group 1 & $12.00 \pm 2.00 \mathrm{~b}$ & $6.99 \pm 2.44 a$ & $4.51 \pm 1.20 b$ & $0.29 \pm 0.12 \mathrm{a}$ & $0.11 \pm 0.10 b$ & $0.11 \pm 0.10 \mathrm{a}$ \\
\hline Group 2 & $19.33 \pm 3.06^{\mathrm{a}}$ & $8.03 \pm 0.20^{\mathrm{a}}$ & $10.73 \pm 3.09^{\mathrm{a}}$ & $0.31 \pm 0.27^{\mathrm{a}}$ & $0.20 \pm 0.22^{\mathrm{ab}}$ & $0.07 \pm 0.12^{a}$ \\
\hline Group 3 & $20.67 \pm 3.06^{\mathrm{a}}$ & $7.60 \pm 0.88^{\mathrm{a}}$ & $12.68 \pm 4.06^{\mathrm{a}}$ & $0.56 \pm 0.18^{\mathrm{a}}$ & $0.49 \pm 0.20^{\mathrm{a}}$ & $0.13 \pm 0.23^{\mathrm{a}}$ \\
\hline \multicolumn{7}{|c|}{ Week 3} \\
\hline Group 1 & $13.33 \pm 1.15^{b}$ & $8.39 \pm 0.71^{\mathrm{a}}$ & $4.26 \pm 0.33^{b}$ & $0.41 \pm 0.16^{\mathrm{a}}$ & $0.23 \pm 0.09^{\mathrm{a}}$ & $0.05 \pm 0.08^{a}$ \\
\hline Group 2 & $21.33 \pm 4.16^{\mathrm{a}}$ & $8.39 \pm 0.23^{\mathrm{a}}$ & $12.59 \pm 4.17^{\mathrm{a}}$ & $0.36 \pm 0.16^{\mathrm{a}}$ & $0.0 \pm 0.0^{\mathrm{a}}$ & $0.0 \pm 0.0^{\mathrm{a}}$ \\
\hline Group 3 & $20.67 \pm 3.06^{\mathrm{a}}$ & $5.23 \pm 0.93^{b}$ & $14.91 \pm 2.64^{\mathrm{a}}$ & $0.34 \pm 0.30^{\mathrm{a}}$ & $0.19 \pm 0.18^{\mathrm{a}}$ & $0.0 \pm 0.0^{\mathrm{a}}$ \\
\hline
\end{tabular}

Means with different superscript letters $(a, b)$ in the same vertical column are significantly different at $p \leq 0.05$. 
Table 6: Some serum biochemical profiles of different groups post gentamicin injection

\begin{tabular}{|c|c|c|c|c|c|c|c|c|c|}
\hline Groups & $\begin{array}{l}\text { AST } \\
(\mu / \mathbf{L})\end{array}$ & $\begin{array}{l}\mathbf{A L T} \\
(\boldsymbol{\mu} / \mathbf{L})\end{array}$ & $\begin{array}{l}\mathbf{A L P} \\
(\boldsymbol{\mu} / \mathbf{L})\end{array}$ & $\begin{array}{c}\text { T.P. } \\
\text { (g/dL) }\end{array}$ & $\begin{array}{l}\text { Albumin } \\
\text { (g/dL) }\end{array}$ & $\begin{array}{l}\text { Globulin } \\
\text { (g/dL) }\end{array}$ & $\begin{array}{c}\mathrm{A} / \mathrm{G} \\
\text { (ratio) }\end{array}$ & $\begin{array}{c}\text { Urea } \\
\text { (mg/dl) }\end{array}$ & $\begin{array}{c}\text { Creatinine } \\
(\mathrm{mg} / \mathrm{dl})\end{array}$ \\
\hline \multicolumn{10}{|c|}{ Day 1} \\
\hline Group 1 & $\begin{array}{c}19.13 \pm \\
4.80^{\mathrm{b}}\end{array}$ & $\begin{array}{c}17.60 \pm \\
3.09^{\mathrm{a}}\end{array}$ & $\begin{array}{c}135.7 \pm \\
68.3^{\mathrm{b}}\end{array}$ & $\begin{array}{l}7.08 \pm \\
0.43^{\mathrm{a}}\end{array}$ & $\begin{array}{l}1.97 \pm \\
0.10^{\mathrm{a}}\end{array}$ & $\begin{array}{c}5.10 \pm \\
0.34^{\mathrm{a}}\end{array}$ & $\begin{array}{c}0.39 \pm \\
0.01^{\mathrm{a}}\end{array}$ & $\begin{array}{l}5.33 \pm \\
2.25^{\mathrm{b}}\end{array}$ & $\begin{array}{l}0.58 \pm \\
0.04^{\mathrm{b}}\end{array}$ \\
\hline Group 2 & $\begin{array}{c}22.40 \pm \\
2.16^{\mathrm{b}}\end{array}$ & $\begin{array}{c}18.77 \pm \\
6.05^{\mathrm{a}}\end{array}$ & $\begin{array}{c}889.5 \pm \\
489.9^{b}\end{array}$ & $\begin{array}{l}4.55 \pm \\
0.85^{b}\end{array}$ & $\begin{array}{l}1.43 \pm \\
0.56^{\mathrm{a}}\end{array}$ & $\begin{array}{l}3.12 \pm \\
0.81^{\mathrm{b}}\end{array}$ & $\begin{array}{c}0.49 \pm \\
0.23^{\mathrm{a}}\end{array}$ & $\begin{array}{c}14.33 \pm \\
0.58^{\mathrm{a}}\end{array}$ & $\begin{array}{l}1.29 \pm \\
0.34^{\mathrm{b}}\end{array}$ \\
\hline Group 3 & $\begin{array}{c}53.80 \pm \\
25.30^{\mathrm{a}}\end{array}$ & $\begin{array}{c}21.55 \pm \\
7.02^{\mathrm{a}}\end{array}$ & $\begin{array}{c}1952.9 \pm \\
688.0^{\mathrm{a}}\end{array}$ & $\begin{array}{l}3.10 \pm \\
1.61^{\mathrm{b}}\end{array}$ & $\begin{array}{l}1.38 \pm \\
0.32^{\mathrm{a}}\end{array}$ & $\begin{array}{l}1.72 \pm \\
1.37^{\mathrm{b}}\end{array}$ & $\begin{array}{l}1.08 \pm \\
0.62^{a}\end{array}$ & $\begin{array}{c}14.67 \pm \\
3.79^{\mathrm{a}}\end{array}$ & $\begin{array}{l}5.15 \pm \\
3.32^{\mathrm{a}}\end{array}$ \\
\hline \multicolumn{10}{|c|}{ Day 3} \\
\hline Group 1 & $\begin{array}{c}11.98 \pm \\
2.34^{\mathrm{b}}\end{array}$ & $\begin{array}{c}13.09 \pm \\
5.11^{\mathrm{b}}\end{array}$ & $\begin{array}{c}116.1 \pm \\
24.7^{c}\end{array}$ & $\begin{array}{l}7.14 \pm \\
1.20^{\mathrm{a}}\end{array}$ & $\begin{array}{l}2.04 \pm \\
0.37^{\mathrm{a}}\end{array}$ & $\begin{array}{l}5.10 \pm \\
1.55^{\mathrm{a}}\end{array}$ & $\begin{array}{c}0.45 \pm \\
0.24^{\mathrm{a}}\end{array}$ & $\begin{array}{l}7.79 \pm \\
2.29^{\mathrm{b}}\end{array}$ & $\begin{array}{l}0.65 \pm \\
0.16^{\mathrm{b}}\end{array}$ \\
\hline Group 2 & $\begin{array}{c}28.88 \pm \\
7.69^{\mathrm{b}}\end{array}$ & $\begin{array}{c}23.24 \pm \\
1.98^{\mathrm{ab}}\end{array}$ & $\begin{array}{c}1046.9 \pm \\
701.2^{\mathrm{b}}\end{array}$ & $\begin{array}{r}4.28 \\
\pm 1.01^{\mathrm{b}}\end{array}$ & $\begin{array}{l}2.09 \pm \\
0.41^{\mathrm{a}}\end{array}$ & $\begin{array}{l}2.18 \pm \\
0.62^{\mathrm{b}}\end{array}$ & $\begin{array}{c}0.98 \pm \\
0.12^{\mathrm{a}}\end{array}$ & $\begin{array}{c}11.43 \pm \\
1.25^{\mathrm{a}}\end{array}$ & $\begin{array}{l}1.82 \pm \\
0.38^{\mathrm{a}}\end{array}$ \\
\hline Group 3 & $\begin{array}{c}72.82 \pm \\
15.61^{\mathrm{a}}\end{array}$ & $\begin{array}{c}29.09 \pm \\
8.45^{\mathrm{a}}\end{array}$ & $\begin{array}{c}1946.1 \pm \\
229.2^{\mathrm{a}}\end{array}$ & $\begin{array}{c}3.63 \\
\pm 0.52^{b}\end{array}$ & $\begin{array}{l}2.03 \pm \\
0.62^{a}\end{array}$ & $\begin{array}{l}1.60 \pm \\
0.63^{\mathrm{b}}\end{array}$ & $\begin{array}{c}1.57 \pm \\
1.18^{\mathrm{a}}\end{array}$ & $\begin{array}{c}11.82 \pm \\
1.66^{\mathrm{a}}\end{array}$ & $\begin{array}{l}2.37 \pm \\
0.85^{\mathrm{a}}\end{array}$ \\
\hline \multicolumn{10}{|c|}{ Week 1} \\
\hline Group 1 & $\begin{array}{c}14.07 \pm \\
6.27^{\mathrm{b}} \\
\end{array}$ & $\begin{array}{c}16.40 \pm \\
3.12^{\mathrm{a}} \\
\end{array}$ & $\begin{array}{r}235.0 \pm \\
743.2^{\mathrm{c}} \\
\end{array}$ & $\begin{array}{c}3.76 \pm \\
0.50^{\mathrm{a}} \\
\end{array}$ & $\begin{array}{c}1.98 \pm \\
0.22^{\mathrm{a}} \\
\end{array}$ & $\begin{array}{l}1.78 \pm \\
0.35^{\mathrm{a}} \\
\end{array}$ & $\begin{array}{l}1.13 \pm \\
0.18^{\mathrm{a}} \\
\end{array}$ & $\begin{array}{c}9.43 \pm \\
1.0^{\mathrm{b}} \\
\end{array}$ & $\begin{array}{l}0.57 \pm \\
0.12^{\mathrm{b}} \\
\end{array}$ \\
\hline Group 2 & $\begin{array}{l}39.09 \pm \\
10.58^{a b}\end{array}$ & $\begin{array}{c}22.49 \pm \\
478^{\mathrm{a}}\end{array}$ & $\begin{array}{c}743.2 \pm \\
75.8^{\mathrm{b}}\end{array}$ & $\begin{array}{c}2.82 \pm \\
0.22^{\mathrm{b}}\end{array}$ & $\begin{array}{c}1.23 \pm \\
0.05^{\mathrm{b}}\end{array}$ & $\begin{array}{c}1.59 \pm \\
0.27^{\mathrm{a}} \\
\end{array}$ & $\begin{array}{c}0.80 \pm \\
0.16^{\mathrm{b}}\end{array}$ & $\begin{array}{c}16.28 \pm \\
3.35^{\mathrm{a}}\end{array}$ & $\begin{array}{c}1.14 \pm \\
0.21^{\mathrm{b}}\end{array}$ \\
\hline Group 3 & $\begin{array}{c}63.00 \pm \\
37.03^{\mathrm{a}}\end{array}$ & $\begin{array}{c}24.43 \pm \\
3.53^{\mathrm{a}}\end{array}$ & $\begin{array}{c}964.7 \pm \\
96.1^{\mathrm{a}}\end{array}$ & $\begin{array}{c}2.16 \pm \\
0.33^{\mathrm{b}}\end{array}$ & $\begin{array}{c}0.90 \pm \\
0.12^{\mathrm{c}}\end{array}$ & $\begin{array}{c}1.27 \pm \\
0.22^{\mathrm{a}}\end{array}$ & $\begin{array}{c}0.71 \pm \\
0.05^{b}\end{array}$ & $\begin{array}{c}17.14 \pm \\
2.57^{\mathrm{a}}\end{array}$ & $\begin{array}{c}2.81 \pm \\
1.20^{\mathrm{a}}\end{array}$ \\
\hline \multicolumn{10}{|c|}{ Week 3} \\
\hline Group 1 & $\begin{array}{c}10.01 \pm \\
1.39^{\mathrm{c}}\end{array}$ & $\begin{array}{c}8.96 \pm \\
2.69^{\mathrm{b}}\end{array}$ & $\begin{array}{c}238.4 \pm \\
53.9^{\mathrm{b}}\end{array}$ & $\begin{array}{c}4.18 \pm \\
0.59^{\mathrm{a}} \\
\end{array}$ & $\begin{array}{c}2.16 \pm \\
0.42^{\mathrm{a}} \\
\end{array}$ & $\begin{array}{c}2.02 \pm \\
0.43^{\mathrm{a}} \\
\end{array}$ & $\begin{array}{l}1.10 \pm \\
0.29^{a}\end{array}$ & $\begin{array}{c}9.05 \pm \\
0.69^{\mathrm{b}}\end{array}$ & $\begin{array}{c}0.58 \pm \\
0.06^{\mathrm{b}} \\
\end{array}$ \\
\hline Group 2 & $\begin{array}{c}19.31 \pm \\
0.86^{\mathrm{b}}\end{array}$ & $\begin{array}{c}17.25 \pm \\
3.31^{\mathrm{a}}\end{array}$ & $\begin{array}{c}656.5 \pm \\
43.4^{\mathrm{a}}\end{array}$ & $\begin{array}{c}2.87 \pm \\
0.65^{\mathrm{b}}\end{array}$ & $\begin{array}{c}1.06 \pm \\
0.18^{\mathrm{b}}\end{array}$ & $\begin{array}{l}1.81 \pm \\
0.48^{\mathrm{a}}\end{array}$ & $\begin{array}{c}0.60 \pm \\
0.08^{b}\end{array}$ & $\begin{array}{c}15.04 \pm \\
1.11^{\mathrm{a}}\end{array}$ & $\begin{array}{l}1.23 \pm \\
0.29^{\mathrm{ab}}\end{array}$ \\
\hline Group 3 & $\begin{array}{c}28.20 \pm \\
4.41^{\mathrm{a}}\end{array}$ & $\begin{array}{c}17.28 \pm \\
2.54^{\mathrm{a}}\end{array}$ & $\begin{array}{c}764.3 \pm \\
81.0^{\mathrm{a}}\end{array}$ & $\begin{array}{c}2.42 \pm \\
0.38^{\mathrm{b}}\end{array}$ & $\begin{array}{c}0.89 \pm \\
0.12^{\mathrm{b}}\end{array}$ & $\begin{array}{l}1.53 \pm \\
0.30^{\mathrm{a}}\end{array}$ & $\begin{array}{c}0.59 \pm \\
0.09^{\mathrm{b}}\end{array}$ & $\begin{array}{c}16.80 \pm \\
1.51^{\mathrm{a}}\end{array}$ & $\begin{array}{c}2.35 \pm \\
1.07^{\mathrm{a}}\end{array}$ \\
\hline
\end{tabular}

Means with different superscript letters $(a, b, c)$ in the same vertical column are significantly different at $\mathrm{p} \leq 0.05$.

Table 7: Levels of GSH and NO in liver and kidney of different groups post gentamicin injection

\begin{tabular}{|c|c|c|c|c|}
\hline Groups & NO Liver & NO Kidney & GSH Kidney & GSH Liver \\
\hline \multicolumn{5}{|c|}{ Week 1} \\
\hline $\begin{array}{l}\text { Control } \\
1^{\text {st }} \text { group }\end{array}$ & $24.13 \pm 0.40^{\mathrm{b}}$ & $16.67 \pm 1.15^{b}$ & $1.36 \pm 1.16^{\mathrm{a}}$ & $3.62 \pm 0.41^{\mathrm{a}}$ \\
\hline $2^{\text {nd }}$ group & $59.73 \pm 0.68^{a}$ & $40.53 \pm 0.65^{a}$ & $1.27 \pm 0.26^{\mathrm{a}}$ & $2.12 \pm 0.13^{b}$ \\
\hline $3^{\text {rd }}$ group & $61.83 \pm 3.18^{\mathrm{a}}$ & $40.77 \pm 1.51^{\mathrm{a}}$ & $1.53 \pm 0.60^{\mathrm{a}}$ & $1.42 \pm 0.43^{b}$ \\
\hline \multicolumn{5}{|c|}{ Week 3} \\
\hline $\begin{array}{l}\text { Control } \\
1^{\text {st }} \text { group }\end{array}$ & $24.40 \pm 0.62^{c}$ & $15.40 \pm 0.72^{b}$ & $2.48 \pm 0.22^{\mathrm{a}}$ & $3.72 \pm 0.41^{a}$ \\
\hline $2^{\text {nd }}$ group & $65.80 \pm 0.20^{b}$ & $45.27 \pm 4.70^{\mathrm{a}}$ & $0.95 \pm 0.08^{b}$ & $1.66 \pm 0.14^{b}$ \\
\hline $3^{\text {rd }}$ group & $71.17 \pm 1.30^{\mathrm{a}}$ & $49.63 \pm 1.01^{\mathrm{a}}$ & $0.82 \pm 0.09^{b}$ & $0.71 \pm 0.13^{c}$ \\
\hline
\end{tabular}

Means with different superscript letters $(a, b, c)$ in the same vertical column are significantly different at $\mathrm{p} \leq 0.05$. 


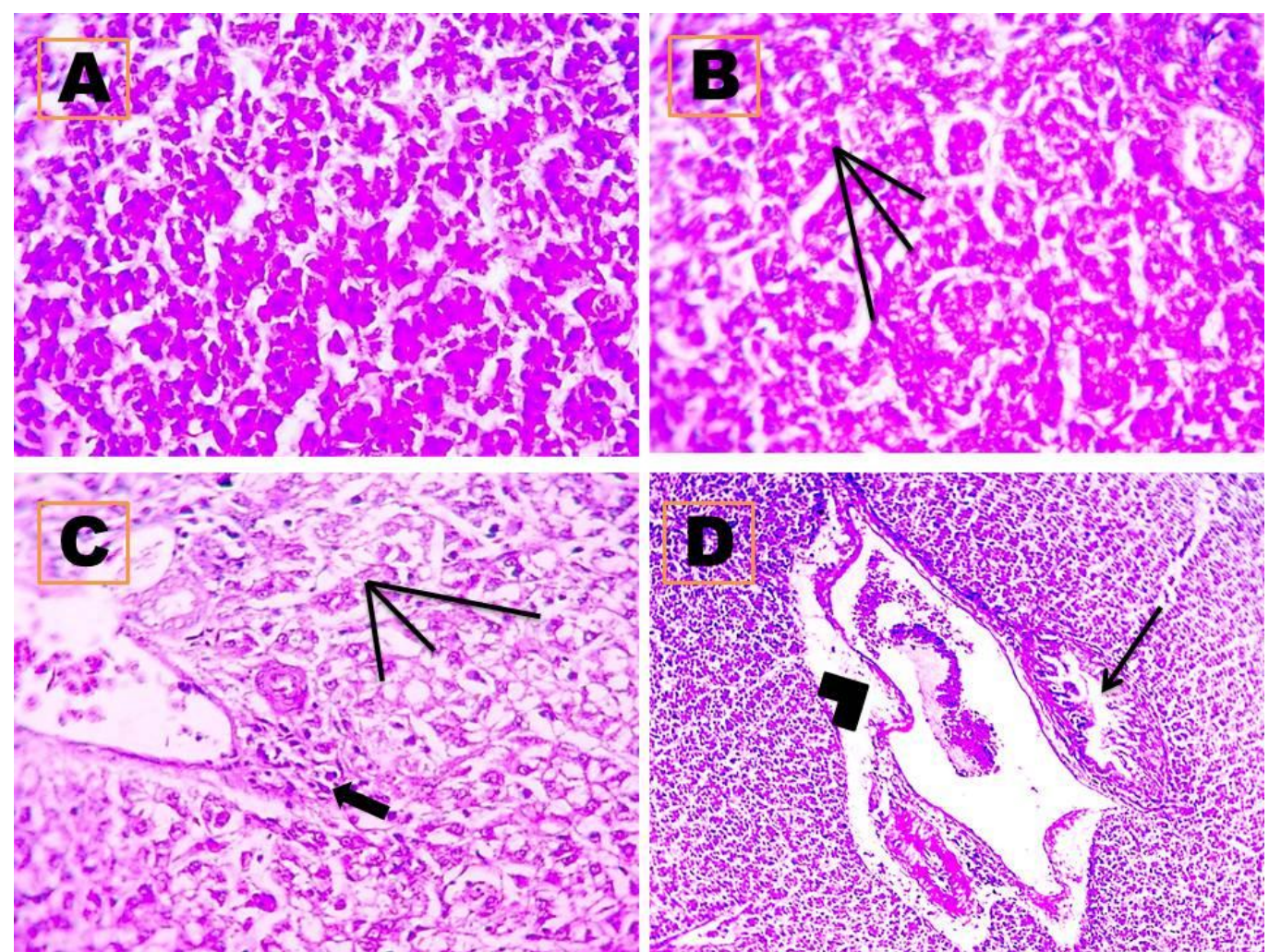

Fig.1: liver shows normal shape and sizes of hepatocytes in Group (1) (A), vacuolar degeneration in hepatocytes with small sized vacuoles (black lines) in Group (2) at $1^{\text {st }}$ week post gentamicin injection (B), diffuse hydropic degeneration in hepatocytes (black lines) with few perivascular leukocytic cells infiltration (arrow) (C), perivascular edema (arrowhead) and dilation of bile duct with hyperplasia of biliary epithelium (arrow) in Group (2) at $3^{\text {rd }}$ week post gentamicin injection (D) (arrow) (H\&E, A- C: x 200 and D: $x$ 100).
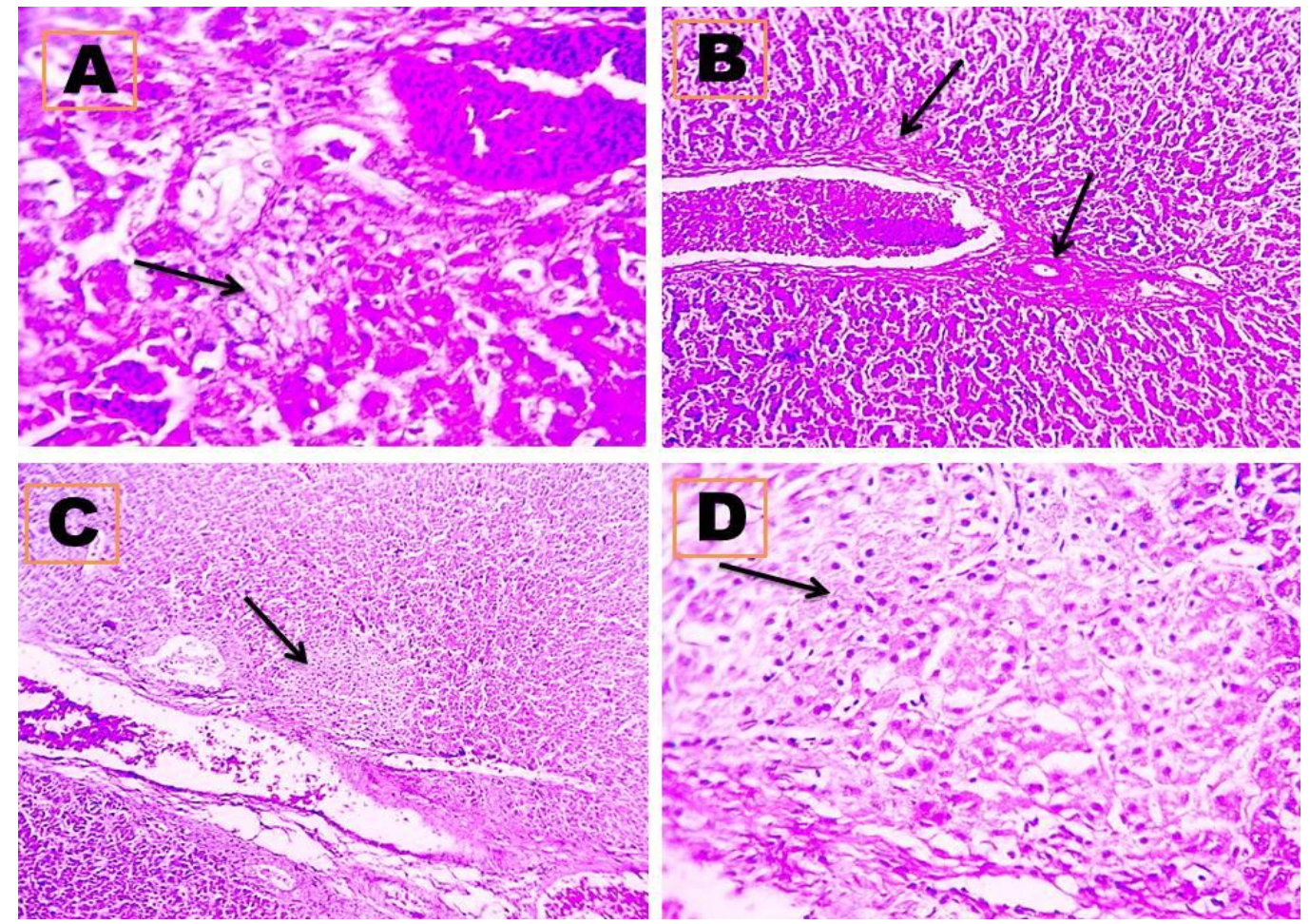

Fig. 2: Liver in Group (3) at $3^{\text {rd }}$ week post gentamicin injection shows new bile ductule formation (arrow) (A), perivascular fibrosis (arrows) (B), focal area of coagulative necrosis (arrow) (C\&D). (H\&E A\&D: x 200, B\&C: $\mathrm{x} 100)$. 

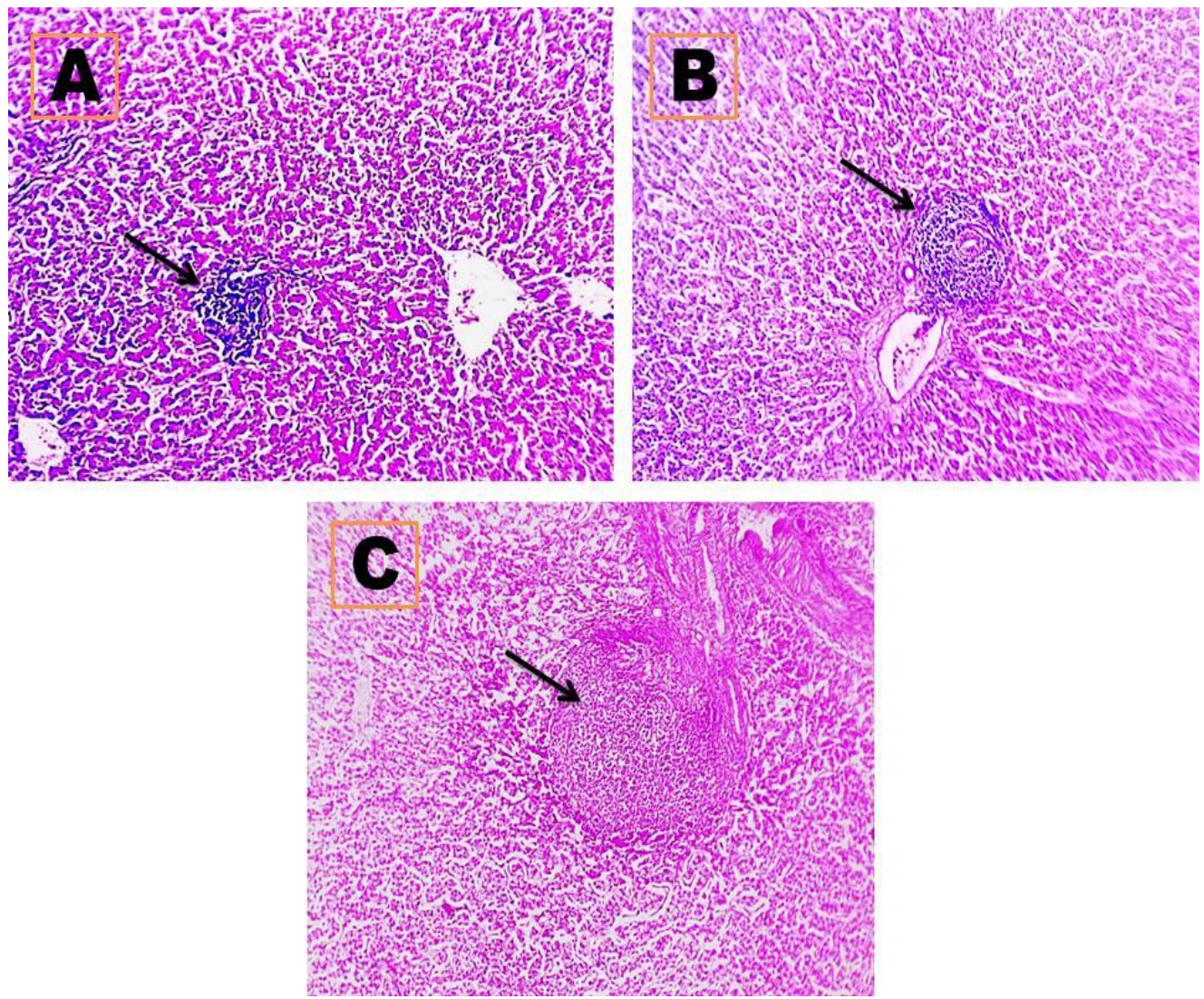

Fig. 3: Liver shows different sizes of lymphocytic aggregation (arrow) (A-C) in Groups (1 to 3), respectively (H\&E A-C: x 100).
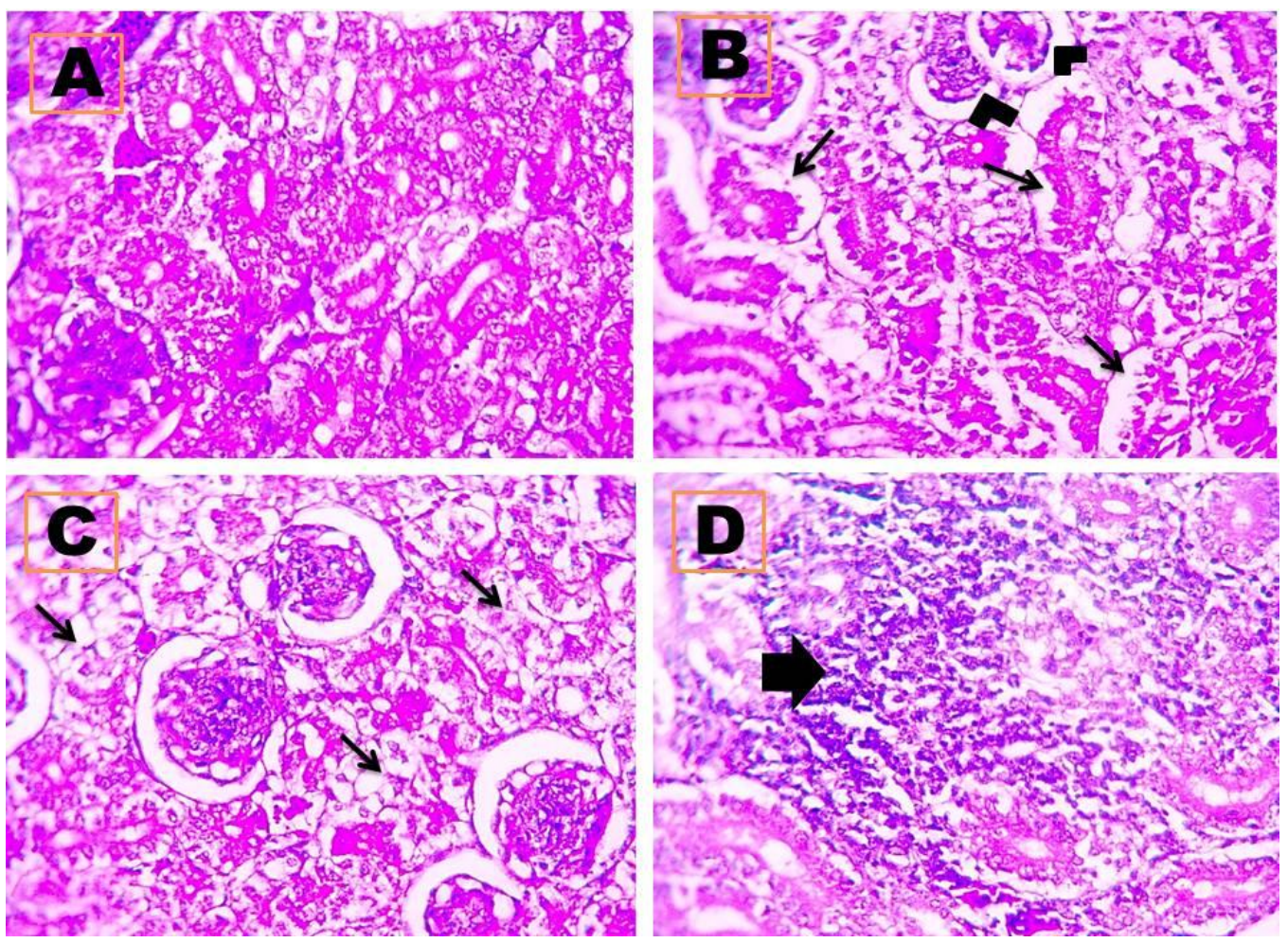

Fig. 4: Kidney shows normal tubules and glomeruli in Group (1) (A), shrunken glomeruli (arrowheads), separation of renal epithelium from basement membrane (arrows) (B), hydropic degeneration in renal tubules (arrows) (C) and focal lymphocytic cells infiltration in interstitial tissue (thick arrow) (D) of Group (2) one week post gentamicin injection (H\&E x 200). 


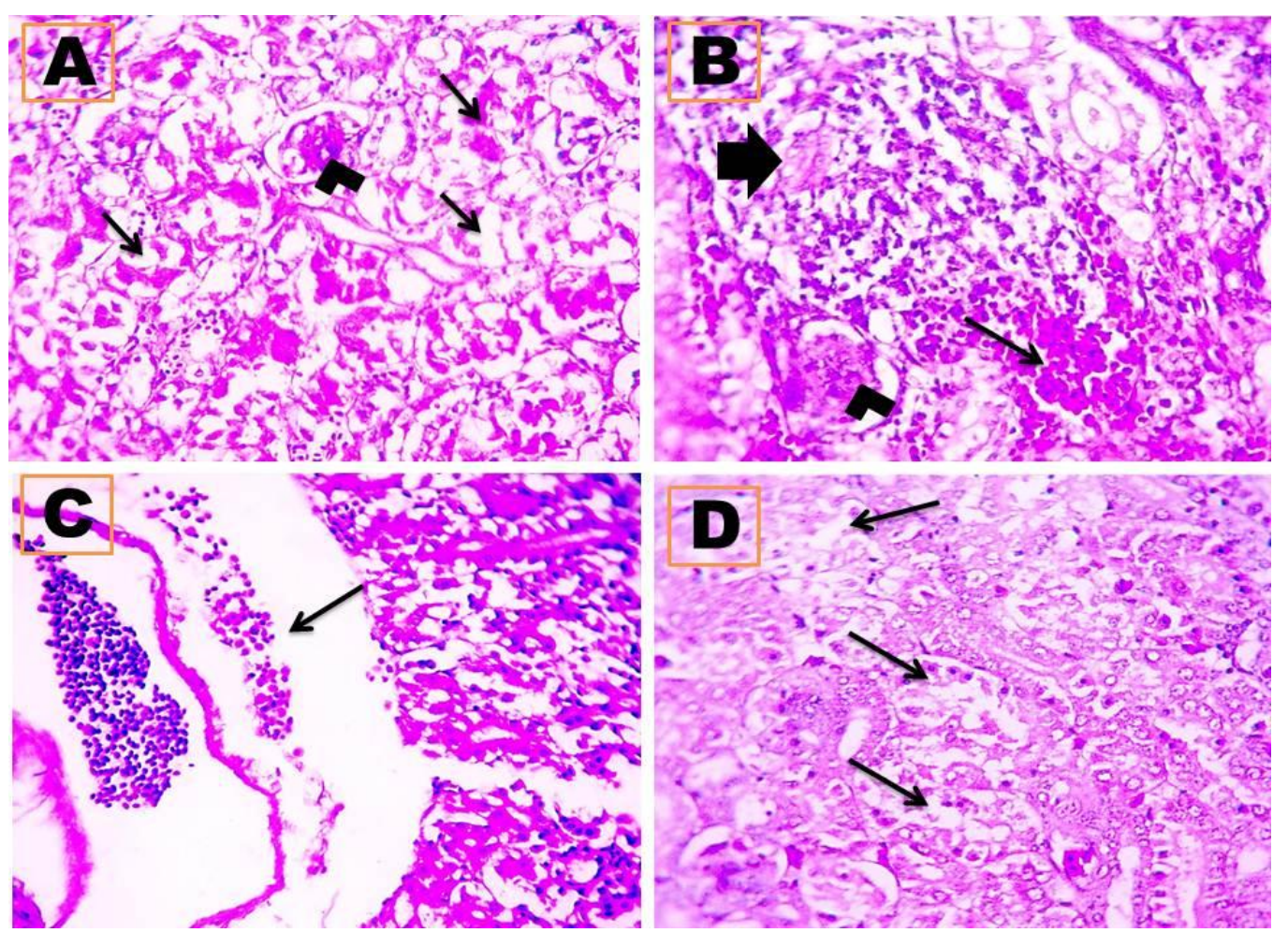

Fig.5: Kidney of Group (3) three weeks post gentamicin injection shows severe hydropic degeneration in renal tubules (arrows), shrunken glomerulus (arrowhead) (A), lymphocytic cells aggregation in renal parenchyma (thick arrow), degenerated glomerulus (arrowhead), hemorrhage (thin arrow) (B), perivascular edema and hemorrhage (arrow) (C) and focal coagulative necrosis in some renal tubules (arrows) (D) (H\&E x 200).

\section{DISCUSSION}

There are many cases of unexplained nephritis; hepatitis and high mortality were reported in poultry farms, which might be due to using the nephrotoxic substances such as gentamicin. Because of the extensive use of gentamicin in our Egyptian poultry farms, we were encouraged to correlate between clinical signs, biochemical alterations and pathological changes induced by gentamicin in chickens.

Chickens administered gentamicin $50 \mathrm{mg} / \mathrm{kg}$ showed a significant decrease in growth performance throughout the experiment and this was due to anorexia and depression following gentamicin administration. Similar results were recorded by Javed et al. (2015) who showed that gentamicin at level 50 $\mathrm{mg} / \mathrm{kg} \mathrm{BW}$ in broilers resulted in severe clinical signs and $33.33 \%$ mortalities. These observations were in line with earlier studies in day old broiler chicks and commercial white leghorn layers (Saleemi et al., 2009). In addition, Islam et al. (2011) observed dehydration, emaciation, watery diarrhea, increased water intake and high mortality in field cases of gentamicin toxicity in White Leghorn layer flocks.
Clinical signs and mortalities in this study were more obvious in injected chickens with gentamicin at level $50 \mathrm{mg} / \mathrm{kg}$ BW suggesting this level was toxic for chickens. The safe dose of gentamicin was $10 \mathrm{mg} / \mathrm{kg}$ BW in day old broilers (Saleemi et al., 2009) and in growing White Leghorn cockerels (Khan et al., 2008).

Normal erythrogram picture indicated normal erythropoitic tissue and adequate requirements (Coles, 1986). Gentamicin in Groups (2\&3) revealed two types of anemia, normocytic normochromic and normocytic hypochromic anemia respectively at $1^{\text {st }}$ week. This anemia changed to macrocytic hypochromic type (after two weeks), which may be attributed to the reduced erythropoietin hormone and enhanced RBC fragility consequence to gentamicin injection (Nagano et al., 1990). The erythropoietin inhibition in the renal cortex altered the basic hematological parameters in rats (Kalaidjieva and Iliev, 2000). In addition, the present uremia inhibited the erythropoiesis and led to intravascular hemolysis (Hostetter and Andreasen, 2004). Moreover, the present heterophilic leukocytosis and eosinophilia indicated the inflammatory response to gentamicin (Irizarry-Rovira et al., 2006). The hematological results were in harmony with results of Saleemi et al. (2009) and established by elevation of NO and depression of GSH levels of the present results. The 
NO had a critical cellular function, besides a potent mediator of cellular damage (Pacher et al., 2007). In this study, gentamicin significantly increased the oxidative stress that was clear from increased total oxidant status in Groups (2\&3) more than in Group (1) agreeing with Haritova et al. (2004); Islam et al. (2011); ELazab and EL-Habashi (2015). Regarding to the gentamicin elimination route, it accumulated in the renal proximal tubular cells through the megalin/cubilin complex receptor, which is responsible for carrying gentamicin inside the cell (Said, 2011). Several studies showed that the ROS involved in gentamicin -induced nephrotoxicity, as well as, it has been shown to enhance the generation of superoxide anion $(\mathrm{O} 2)$, peroxynitriteanion (ONOO), hydrogen peroxide $(\mathrm{H} 2 \mathrm{O} 2)$, and hydroxyl radical $(\mathrm{OH})$ from renal cortical mitochondria, which is responsible for increase lipid peroxidation and decrease antioxidant enzymes (Tavafi and Ahmad, 2011). Regarding to the effect of gentamicin on the tested biochemical parameters, this study revealed an elevation in levels of AST, ALT, ALP, urea, creatinine and lowering in total protein, albumin and $\mathrm{A} / \mathrm{G}$ ratio while globulin was normal. The decreased $A / G$ ratio was related to inflammation nephropathy and liver damage caused by gentamicin injection. However, AST, ALT and ALP enzymes were not specific for liver disease in birds but they increased with liver damage (Harr, 2002). Liver enzymes indicated the alteration in cell membrane integrity or biliary epithelium (Eran et al., 2007). Urea was a nitrogenous waste product formed in the liver and execrated via kidneys (Kaneko, 2008). Albumin was formed in liver, lost in glomerulonephritis but the globulin could be normal. Hypoalbuminemia was an independent predictor for acute renal injury and death. Creatinine investigated kidney disease and could not influence by liver or urea cycle (Harr, 2002). Many previous reports showed the decrease in TP and albumin levels and increase in ALT and creatinine levels in chicken injected with gentamicin (Saleemi et al., 2009). Its effect extended to cause severe nephrotoxic and hepatotoxic effects (Javed et al., 2015). Generally, gentamicin as all aminoglycosides induced tubular nephrotoxicity (Khan et al., 2008). This may be because gentamicin after removal from the blood accumulated in renal tubular cells and produced phospholipidosis (Beauchamp et al., 1991).

Microscopically, injection of gentamicin induced pathological lesions in growing cockerels (Kaneko, 2008) and growing broilers (Khan et al., 2008). Our results revealed severe degenerative and necrotic changes accompanied with multifocal lymphocytic inflammation were observed in liver and kidneys from chicken injected with $50 \mathrm{mg}$ gentamycin $/ \mathrm{kg}$ BW. Kidney and liver injuries followed by gentamicin administration could be the major reasons for anorexia, decrease in growth performance and subsequent emaciation of the birds. These data agreed with previous findings obtained by Sundin et al. (2001) who mentioned that gentamicin caused inhibition of protein synthesis, resulting in proximal tubule cell necrosis in renal cells in 13-30\% of treated patients. This mechanism specifically caused proximal tubule cells necrosis, resulting in acute tubular necrosis that can lead to acute renal failure.

\section{Conclusion}

Gentamicin administration reduced growth performance, altered hematological, biochemical and serum antioxidants pictures and induced hepatic and renal lesions that appeared to be dose dependent. The chicken administered $50 \mathrm{mg} / \mathrm{kg}$ BW gentamicin as a single IM dose was more severely affected than 25 $\mathrm{mg} / \mathrm{kg} \mathrm{BW}$ gentamicin.

\section{Conflict of interest}

The authors declare that there is no conflict of interest.

\section{Funding sources}

This research did not receive any specific grant from funding agencies in the public, commercial, or notfor-profit sectors.

\section{Abbreviations:}

BW, Body weight; IM, intramuscular; NO, Nitric oxide; GSH, reduced glutathione; ROS, Reactive Oxygen Species; FCR, Feed conversion ratio; RBC, Red blood cells; TLC, total leukocytic count; Hb, hemoglobin; PCV, packed cell volume; MCV, mean corpuscular volume; $\mathrm{MCH}$, mean corpuscular hemoglobin; MCHC, mean corpuscular hemoglobin concentration; A/G, albumin/globulin; O2, superoxide anion; ONOO, peroxynitriteanion; $\mathrm{H} 2 \mathrm{O} 2$, hydrogen peroxide; $\mathrm{OH}$, hydroxyl radical; EDTA, ethylene diaminetetra acetic acid; ALP, alkaline phosphatase; AST, aspartate aminotransferase; ALT, alanine aminotransferase.

\section{Acknowledgment}

We acknowledge support of Departments; Poultry diseases, Pharmacology, Pathology, Physiology and Clinical Pathology, Mansoura University for providing chemicals, instruments and devices.

\section{Author contribution}

Nagah Arafat: putting the study plan, running the experimental schedule, measuring feed and growth parameters, collecting samples and sharing in writing. Reham El-Shafei: sharing the study plan, sharing collecting samples, sharing in writing, revising and submission of the article as a corresponding author. Walaa Awadin: histopathological evaluation, sharing in writing and revising of the manuscript. Verginia Farag: Sharing in collecting samples, writing, revision and measuring serum biochemical parameters, leukogram and erythrogram. Rasha Saleh: measuring antioxidant parameters 


\section{REFERENCES}

Andreasen, CB. and Latimer, KS. (1990): Separation of avian heterophils from blood using FicollHypaque discontinuous gradients. Avian Dis. 33, 163-167.

Bancroft, JD. and Gamble, M. (2007): Theory and Practice of Histological Techniques. 5th Ed; Churchill Livingstone, London, UK, pp: 125138.

Beauchamp, D.; Gourde, P. and Bergeron, MG. (1991): Subcellular distribution of Gentamicin in proximal tubular cells. Antimicrob. Agents Chemother. 35: 2173-2179.

Beutler, E.; Duron, O. and Kelly, BM. (1963): Improved method for the determination of blood glutathione. J. lab. Clin. Med. 61: 882888.

Coles, EH. (1986): Veterinary Clinical Pathology Forth edition W.B. Saunders Co. Philadelphia. 40, pp: 222-225.

Dein, FJ. (1984): Laboratory Manual of Avian Haematology. Association of Avian Veterinarian, East north port, USA.

ELazab, MF. and EL-Habashi, N. (2015): Gentamicin-induced nephrotoxicity in chickens: Modulatory role of Moringa oleifera. Assiut Vet. Med. J. 61 (144).

Eran, E.; Galia, P.; Rifaat, S. and Orit, P. (2007): Association between consumption of Herbalife ${ }^{\circledR}$ nutritional supplements and acute hepatotoxicity. J. Hepatol. 47: 514-520.

Feldman, BF.; Zinkl, JG.; Jain, NC. and Gasper, P. (2000): Schalm's Veterinary Hematology. Wiley.

Haritova, AM.; Djeneva, HA.; Lashev, L.; Sotirova, PG.; Gurov, BI. and Dyankov, VN. (2004): Pharmacokinetics of gentamicin and apramycin in turkeys roosters and hens in the context of pharmacokineticpharmacodynamic relationships. J. Vet. Pharm. Ther. 27:381-384.

Harr, KE. (2002): Clinical chemistry of companion avian species: a review. Vet. Clin. Pathol. 31, 140-151.

Hostetter, SJ. and Andreasen, CB. (2004): General Principles. In: COWELL, R.L. Veterinary clinical pathology secrets. Missouri: Elsevier, pp: $1-3$.

Ignarro, LJ.; Buga, GM.; Wood, KS.; Byrns, RE. and Chaudhuri, G. (1987): Endothelium-derived relaxing factor produced and released from artery and vein is nitric oxide. Proc. Natl. Acad. Sci. USA 84: 9265-9269.

Irizarry-Rovira, AR.; Wolf, A. and Ramos- Vara, JA. (2006): Cutaneous melanophoroma in a green iguana (Iguana iguana). Vet. Clin. Pathol. 35: 101-105.

Islam, NU.; Khan, MZ.; Khan, MK.; Saleemi, A.; Khan, SA.; Bhatti, M.; Yousaf, M. and
Hassan, ZU. (2011): Clinicopathological studies on gentamicin toxicity in White Leghorn commercial layers. Pak. Vet. J. 31: 305-308.

Javed, S.; Khan, JA.; Khaliq, K.; Javed, I. and Abbas, RZ. (2015): Experimental evaluation of nephroprotective potential of Calotropis procera (Ait) flowers against Gentamicin induced toxicity in albino rabbits. Pak. Vet. J. 35(2): 222-226.

Javed, U.; Khan, MZ.; Saleemi, MK.; Khan, A.; Javed, I. and Rafique, S. (2013): ToxicoPathological effects of Parenteral administration of gentamicin in growing broilers. Int. J. Agric. Biol., 15: 529-534.

Javed, U.; Saleemi, MK.; Khan, MZ.; Khan, A. and Javed, I. (2008): Pathological effects of Gentamicin in growing broilers. Proc 15th Congress of FAVA-OIE Joint Symposium on Emerging Diseases, pp: 17-18.

Kalaidjieva, VC. and Iliev, ZK. (2000): Plasma erythropoietin level in rats after kidney proximal tubular impairment. Folia Med. 42: 41-45.

Kaneko, JJ. (2008): Hepatic function. In: JJ Kaneko, JW Harvey and ML Bruss (eds), Clinical Biochemistry of Demostic Animals, $6^{\text {th }}$ Edition, (EAcedemic Press, London), pp: 356365.

Kaneko, JJ.; Harvey, JW. and Bruss, ML. (1997): Clinical biochemistry of domestic animals, $4^{\text {th }}$ Edition.

Kang, C.; Lee, DY.; Hah, JH.; Heo, CH.; Kim, E. and Kim, JS. (2013): Protective effects of Houttuynia cordata Thunb on gentamicin induced oxidative stress and nephrotoxicity in rats. Toxicol. Res. 29: 61-67.

Khan, I.; Khan, MZ.; Saleemi, MK.; Javed, I. and Khan, A. (2008): Pathological and biochemical effects of intramuscular gentamicin administration in chicken. Turk. J. Vet. Anim. Sci. 32: 345-351.

Kovacs, E.; Savopol, T.; Iordache, MM.; Saplacan, L.; Sobaru, I.; Istrate, C.; Mingeot-Leclercq, MP. and Moisescu, MG. (2012): Interaction of gentamicin polycation with model and cell membranes. Bioelectrochem. (Amsterdam, Netherlands) 87: 230-235.

Morales, AI.; Detaille, D.; Prieto, M.; Puente, A.; Briones, E.; Arévalo, M.; Leverve, X.; LópezNovoa, JM. and El-Mir, MY. (2010): Metformin prevents experimental Gentamicin - induced nephropathy by a mitochondriadependent pathway. Kidney Int. 77: 861-869.

Nagano, K.; Kamiyama, T.; Nakao, M. and Nakao, K. (1990): A direct relationship between adenosine triphosphate-level and in vivo viability of erythrocytes. Nature 194:87787852 . 
Natt, MP. and Herrick, CA. (1952): A new blood diluent for counting erythrocytes and leucocytes of the chicken. Poultry Sci. 31, 735-738.

Pacher, P.; Beckman, JS. and Liaudet, L. (2007): Nitric oxide and peroxynitrite in health and disease. Physiol. Rev. 87, 315-424.

Ravi, K.; Vamsi, K. and Boobalan, G. (2015): Adverse effects of routinely used drugs in animal husbandry practice. Eggers $P$. Prevalence of chronic kidney disease in the United States. J. Am. Med. Assoc. 298(17): 2038-47.

Said, MM. (2011): The protective effect of eugenol against gentamicin induced nephrotoxicity and oxidative damage in rat kidney. Fundam. Clin. Pharmacol. 25:708-716.

Saleemi, MK.; Khan, MZ.; Khan, A. and Javed, I. (2009): Pathological effects of gentamicin administered intramuscularly today old broiler chicks. Exp. Toxicol. Path. 61: 425-432.
SAS Institute (2004): SAS Users Guide Statistics version, SAS Institute Inc., Cary, NC-USA.

Sener, G.; Sehirli, A.; Altunbas, O.; Ersoy, HZ.; Paskaloglu, Y.; Arbak, K. and AyanogluDulger, G. (2002): Melatonin protects against gentamicin-induced nephrotoxicity in rats. $\mathrm{J}$. Pineal Res. 32, 231-236.

Smith, T. (1999): Commercial broiler production. Mississippi State University, Mississippi State, MS. Chapter 2, pp: 7-13.

Sundin, DP.; Sandoval, R. and Molitoris, BA. (2001): Gentamicin inhibits renal protein and phospholipid metabolism in rats: implications involving intracellular trafficking. J. Am. Soc. Nephrol. 12:114-123.

Tavafi, M. and Ahmad, H. (2011): Effect of rosmarinic acid on inhibition of gentamicin induced nephrotoxicity in rats. Tissue Cell 43:392-397.

\section{عواقب التسمم الكبدى الكلوي بالجنتاميسين في الاجاج}

نجاح عرفات ، ريهام أحمد الشافعي ، ولاء فكرى ، فيرجينيا محمد المتولح ، رشا محمد صالح

Email: Dr_reham16@yahoo.com Assiut University web-site: www.aun.edu.eg

يستخدم الجنتاميسين، كأحد الأمينوجلوكوسيدات في علاج الالتهابات البكتيرية المختلفة في الحيوان. لقد أجريت هذه الدراسة لبحث

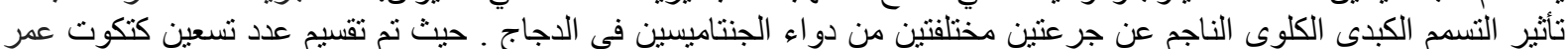

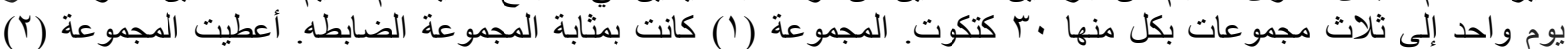

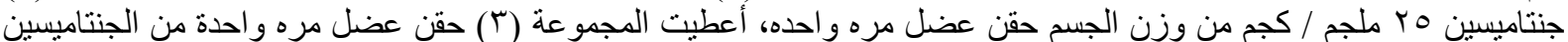

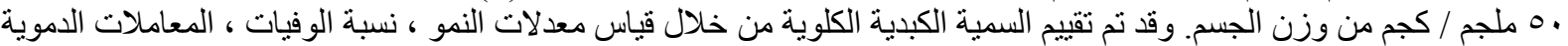

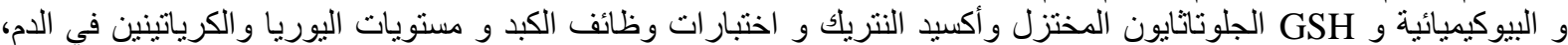

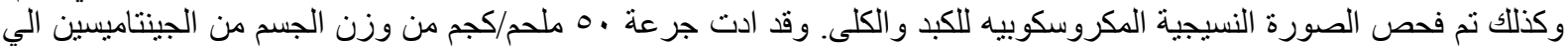

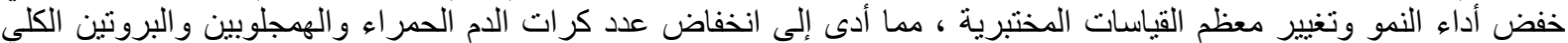

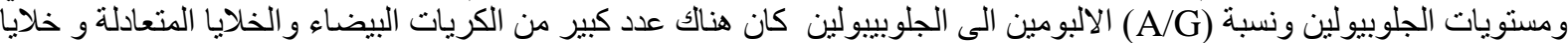

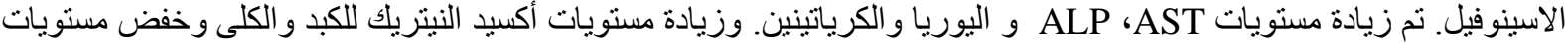

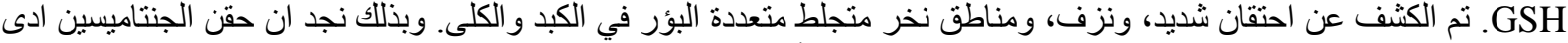

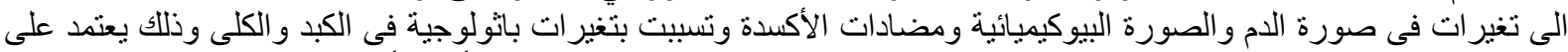

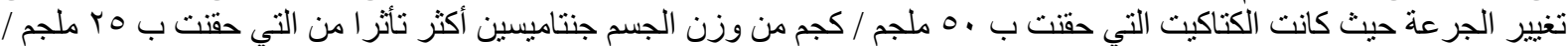
كجم من وزن الجسم جنتاميسين. 